\title{
Automatic Segmentation of Prostate Cancer using cascaded Fully Convolutional Network
}

\author{
Padmavathi Kora ${ }^{1, *}$, Reddy Madhavi $\mathrm{K}^{2}$, Avanija $\mathrm{J}^{2}$, Sunitha Gurram ${ }^{2}, \mathrm{~K}_{\text {Meenakshi }}{ }^{1}$, K Swaraja ${ }^{1}$, Y Priyanka ${ }^{1}$ \\ ${ }^{1}$ GRIET, Hyderabad, India \\ ${ }^{2}$ Sree Vidyanikethan Engineering College, AP, India
}

\begin{abstract}
In this paper we proposed a prostate segmentation and also tumour detection using deep neural networks. The cutting-edge deep learning techniques are useful compared to the challenges of machine learning based feature extraction techniques. Here we proposed a strategy that contains an FCN model that incorporates data from several MRI images, allowing for faster convergence and more accurate segmentation. T1 and DWI volumes may be used together to delineate the prostate boundary, according to this study. Second, we investigated whether this method might be utilized to provide voxel-level prostate tumor forecasts. The cascaded learning method and performed tests to demonstrate its effectiveness. Keywords: Prostate cancer, FCN, MR images, CNN.
\end{abstract}

\section{INTRODUCTION}

Prostate cancer has been major cause of death in the US, therefore early identification and diagnosis are critical for lowering mortality and enhancing treatment success. The procedures for cancer detection are intrusive and have a high risk of false positives. Alternatively, MRI is a imaging method that allows for more accurate prostate cancer detection and diagnosis. However, reading and interpreting these pictures by radiologists is typically challenging owing to the huge number of 3D MR slices and the reading procedure being laborious and time consuming. As a result, in the last two decades, creating computer-based systems for automated diagnosis has piqued researchers' attention. The prostate region is first segmented from the background tissues, and subsequently lesions inside the prostates are detected using such CAD techniques.

Traditional machine learning-based CAD systems [6],

[7], [8] for prostate cancer diagnosis typically use a twostep architecture that includes a candidate generation step and a false-positive reduction step. For example, Litjens et al., [1] generated region-based statistical characteristics for candidate classification after extracting voxel-based characteristics for candidate creation. For both phases, linear discriminant classifier and ensemble learning models were used. A lot of recent research has looked at using deep learning approaches to solve the detection problem. For pixel-level prediction, Tsehay et al. [2] used a holistic layered network with deep supervision technique. Stacking auto-encoders and a random forest were utilised by Zhu et al., [3]. To increase detection performance, Kohl et al., [4] presented a network design that included an FCN and an adversarial network.

The viability of establishing a novel prostate cancer detection technique based on a deep neural network model is being investigated in this research. The suggested approach comprises of two networks, one for prostate segmentation and the other for tumour identification. Because it can be trained and give fast pixel level detection, the fully convolutional network (FCN) architecture is used for both processes. Since a number of recent research has established the efficiency of mpMRI-based CAD techniques, from mpMRI images. for both prostate area segmentation and lesion diagnosis.

The motivation for conducting this study is twofold: (1) we are able to introduce a FCN to focus on the hurdles of cancer segmentation and detection, and (2) to apply mpMRI for prostate area segmentation, which is different from literature that only worked on $\mathrm{T} 2 \mathrm{~W}$ based segmentation. As a result, we conducted the following tests using a prostate MR dataset to assess the efficacy of prostate area segmentation and tumour identification using our suggested technique.

\section{PROSTATE SEGMENTATION BASED ON MPMRI}

\section{1 Pre-processing and data set}

The prostate segmentation step was developed using data from images of 195-people. A total of 160,35 instances are used for training and testing at random. Multiparametric modalities with the greatest b-value were found for each instance, including T2W, T1, and DWI. An expert radiologist used $\mathrm{T} 2 \mathrm{~W}$ pictures to identify the

\footnotetext{
* Corresponding author: padma386@gmail.com
} 
masks of prostatic regions as ground truth. The ground truth masks and volumes of interest were scaled to a fixed spacing of 11.3 millimetres. For each MR imaging modality, a 3D patch of 1281284 was clipped, and the voxel values were standardized (0-1 variance).

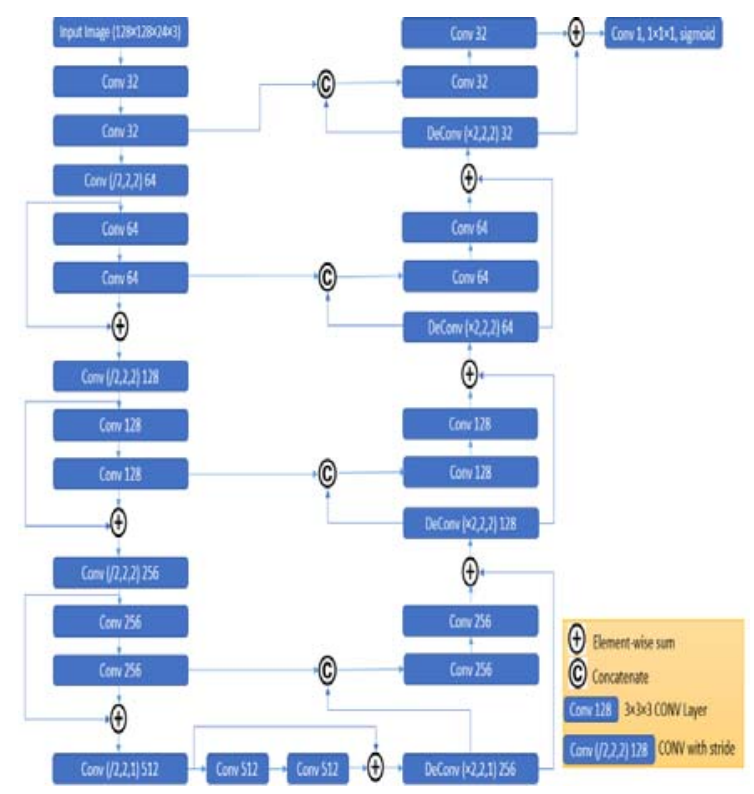

Fig. 1. The FCN architecture

\subsection{Networking architecture}

The design of 3D designs like Milletari et al., [5] shown in Figure 1. The FCN receives images with dimensions consisting of input altitude, breadth, channel and deepness. A decreasing left and increasing right routes make up the network. In the contraction approach, 333 conventional layers and ReLU activation function to get high-feature information. To receive more spatial information, CNNs with steps larger than one are used with step by step decrease breakdown while expanding the previous layer are used. Input signals are instantly integrated into output feature maps at each resolution level, enabling the well-stacked convolution layers. The acquiring route produces feature-maps with poorresolution, whereas the expanding route samples featuremaps that produce dense-prediction. Deconvolution techniques can be used to enhance feature map breakdown. To incorporate high-resolution information, feature-maps produced are combined with deconvolutionary layer end products. The expanded route also uses residual connections. A convolutionary layer with 111 kernels and sigmoid activation function processes the output feature-maps with the same dimension as the input-images. During the trainingphase, the goal is to find the dice-coefficient. The initial segmentation produced by $\mathrm{FCN}$ is subsequently refined using post-processing methods. We utilised a 3D Gaussian filter and connected component analysis to eliminate tiny isolated components and to smooth the output maps.

\subsection{Evaluation and implementation}

It was developed using the Tensorflow library. During the training phase, we utilised an augmentation method to produce more data. The network was trained using the Adam optimizer and the weights are random. The optimised network used the prostate images in the testing set as input and conducted a back propagation computation for testing. The post-processing stages adjust the resulting probability maps before thresholding. For performance evaluation, dice output values are computed between predicted output and ground-truth output.

\section{DETECTION OF CANCER}

The input images are obtained with different inquisition formats, such as DWI and T2W, of 79 individuals utilised to construct the tumour identification step. DWI was used to create ADC maps, and dynamic-contrast enhanced MR-perfusion was used to create K-trans pictures. Radiologists discovered at least one tumour in each patient, and the tumour area's perimeter was also noted.

A total of 60 instances of the training-set and a testingset of 19 instances were randomly assigned to the dataset. We used a 2D network for tumour identification instead of a segmentation step since the sizes of indicated tumours are rather tiny in comparison to the vertical resolution. The network was trained on data collected from 60 subjects with 500 slices. There were malignancies in 260 slices and no tumours in the remaining 140 slices. The prostate segment region is clipped and computed to 176176 images from each slice. The pixel values were normalised using the Z-score method. We used random shift, rotation, and scaling to increase the training set.

\subsection{Networking design}

The FCN employed for tumour extraction is shown in Figure 2. Because both networks try to provide voxellevel predictions, they are comparable to the previous networks. We use a somewhat different architecture with three down sampling layers in the getting route due to the limitations of annotated data. The network accepts two-dimensional pictures with four channels as input and generates tumour masks. To train the network, the goal function is weighted cross-entropy loss. For each single voxel, voxel-level cross entropy loss was estimated first. To construct the objective function, a hyper-parameter $\mathrm{w}$ multiplied the losses of positive voxels and then added negative voxel losses. We may change weights between positive and negative predicted losses by changing $\mathrm{w}$. We also utilise L2 to avoid overflow. 


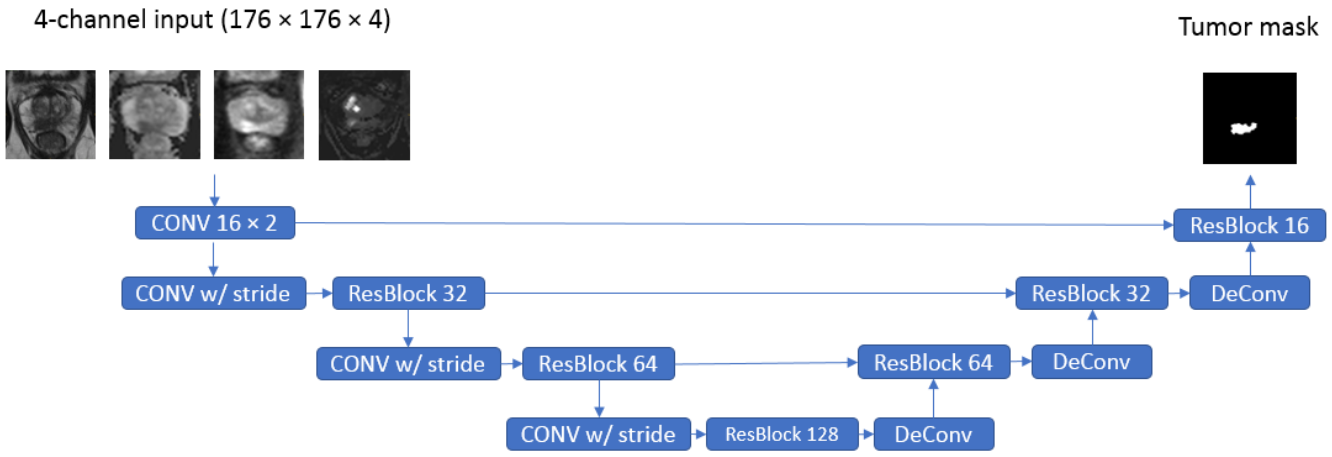

Fig. 2. The FCN architecture based tumor detection

\subsection{Evaluation and Implementation}

To train the network, we used a hybrid technique. To begin, we trained the FCN with large-epochs using a big $\mathrm{w}$ value. The goal is to have a quick first detection with a high sensitivity. We computed the positive voxels created by the first detection and trained the network with a reduced $\mathrm{w}$ value. We supplied image slices into the network during the testing phase and layered 2D predictions to get $3 \mathrm{D}$ detection. To test the suggested detection approach, we drew a ROC-curve at various levels. The classification rate between ground-truth masks and predicted-masks. A successful detection is defined as more than $10 \%$--voxels of a tumour being labelled as positive-images and the false-positive-rate is also computed.

\section{CLASSIFICATION RESULTS}

For segmentation, we used 100-epochs and trained on the FCN-network. We have created a second FCN with the same design, but this one only accepts $\mathrm{T} 2 \mathrm{~W}$ image data as input. The performance of the FCN-network is summarised in Table 1. It indicates that incorporating information from several MR modalities improves segmentation performance when compared to simply utilising T2W image data. mpMRI-based FCN, on the other hand, produces quicker convergence than $\mathrm{T} 2 \mathrm{~W}$ based FCN.

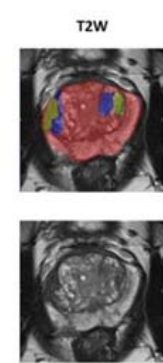

T2W
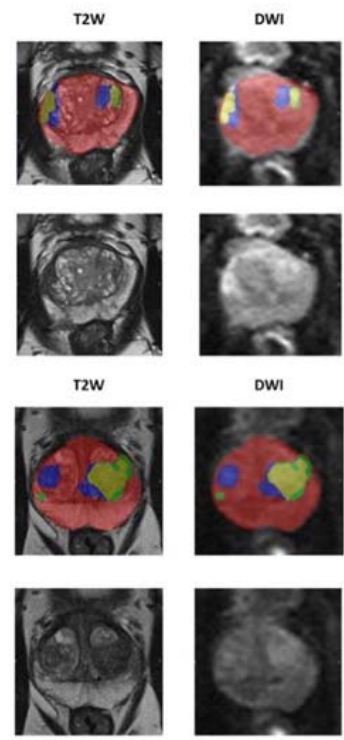

ow!
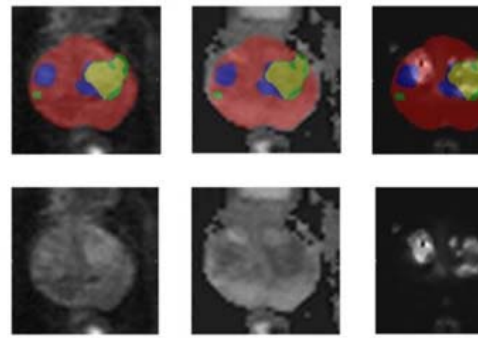

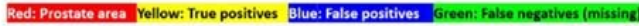

Fig.3. FCN detection results with cascaded training

Table 1: FCN segmentation-performance

\begin{tabular}{ccc}
\hline FCN-input & $\begin{array}{c}\text { Training- } \\
\text { epochs }\end{array}$ & $\begin{array}{c}\text { Average-Dice- } \\
\text { coefficient }\end{array}$ \\
\hline T2W & 2000 & 0.8818 \\
T2W, DWI, & 500 & 0.8935
\end{tabular}

$\mathrm{T} 1$

The red performance curve is acquired by training the network using the first stage, whereas the blue performance curve is acquired by using the two-stage training technique. It demonstrates that a cascadedtraining approach improves detection performance, yielding a $100 \%$ classification rate with a false-positive

\footnotetext{
*Corresponding author: padma386@gmail.com
} 
rate of less than 0.2. Figure 3 illustrates two instances of detection findings obtained with a detection rate of 0.85 .

The single-stage training method is represented by the red line, while the cascaded training method is shown by the blue line. Two examples of classification accuracies achieved by the FCN network are shown in Figure 4. Two successful detections are shown in the top case, whereas one eminent detection, one false positive, and one undiscovered tumour.

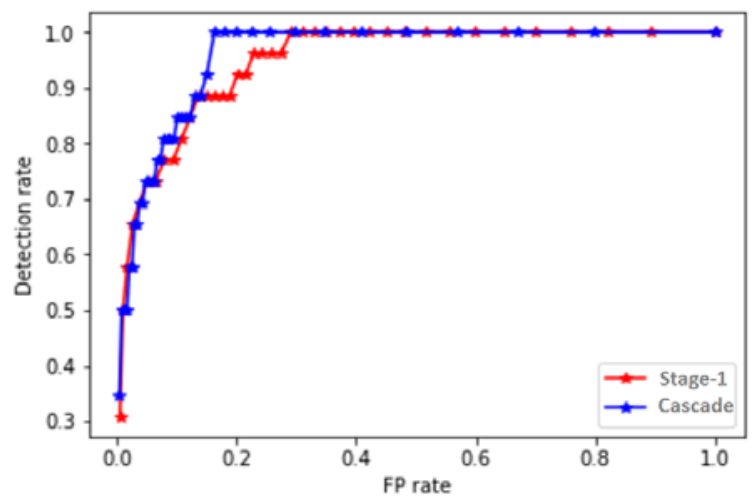

Fig. 4. Performance using ROC curve.

\section{CONCLUSION}

We designed and evaluated a CAD system for prostate cancer detection based on mpMRI in this work. To overcome the difficulties of hand-crafted feature extraction, we used cutting-edge deep learning models. The suggested plan has a number of distinguishing features. Alternatively, to using a single $\mathrm{T} 2 \mathrm{~W}$ segmentation modality, we designed an FCN network that integrates image data from several MR modalities, enabling accurate segmentation. It indicates that $\mathrm{T} 1$ and DWI images may be used in conjunction to detect prostate cancer. Second, we looked at whether a proposed network architecture might be used to create voxel-level predictions for tumour identification. We suggested a cascaded-training technique and conducted trials to illustrate its efficacy. Despite the positive findings, this is preliminary research with a limited sample size. In the future, the performance of the suggested CAD technique will need to be tested using a bigger dataset.

\section{References}

[1] G. Litjens, O. Debats, J. Barentsz, N. Karssemeijer, H. Huisman, Computer-aided detection of prostate cancer in mri, IEEE transactions on medical imaging 33 (5) (2014) 1083-1092.

[2] Y. K. Tsehay, N. S. Lay, H. R. Roth, X. Wang, J. T. Kwak, B. I. Turkbey, P. A. Pinto, B. J. Wood, R. M. Summers, Convolutional neural network based deep learning architecture for prostate cancer detection on multiparametric magnetic resonance images, in: Medical Imaging 2017: Computer-Aided Diagnosis, Vol.

10134, International Society for Optics and Photonics, 2017, p. 1013405.

[3] Y. Zhu, L.Wang, M. Liu, C. Qian, A. Yousuf, A. Oto, D. Shen, Mri-based prostate cancer detection with high-level representation and hierarchical classification, Medical physics 44 (3) (2017) 1028-1039.

[4] S. Kohl, D. Bonekamp, H.-P. Schlemmer, K. Yaqubi, M. Hohenfellner, B. Hadaschik, J.-P. Radtke, K. MaierHein, Adversarial networks for the detection of aggressive prostate cancer, arXiv preprint arXiv:1702.08014.

[5] F. Milletari, N. Navab, S.-A. Ahmadi, V-net: Fully convolutional neural networks for volumetric medical image segmentation, in: 2016 fourth international conference on 3D vision (3DV), IEEE, 2016, pp. 565571.

[6] Kuraparthi, Swaraja, Meenakshi Kollati, and Padmavathi Kora. "Robust Optimized Discrete Wavelet Transform-Singular Value Decomposition Based Video Watermarking." Traitement du Signal 36.6 (2019).

[7] Kora, Padmavathi, and K. Sri Rama Krishna. "Bundle block detection using genetic neural network." Information systems design and intelligent applications. Springer, New Delhi, 2016. 309-317.

[8] Kora, Padmavathi, K. Meenakshi, K. Swaraja, A. Rajani, and Md Kafiul Islam. "Detection of cardiac arrhythmia using fuzzy logic." Informatics in Medicine Unlocked, 17, 2019, pp. 100257. 